\title{
Wave-packet dynamics in quantum spin Hall systems
}

\author{
Marcos H. L. de Medeiros ${ }^{1}$, Gerson J. Ferreira ${ }^{2}$, Luis G. Dias da Silva ${ }^{3}$ \\ University of São Paulo ${ }^{1,3}$, Federal University of Uberlândia ${ }^{2}$ \\ marcos.henrique.medeiros@usp.br',gersonjferreira@ufu.br ${ }^{2}$, luisdias@if.usp.br ${ }^{3}$
}

Keywords: topological insulators, spin hall, zitterbewegung

\section{Abstract}

In the context of research on topological phases of matter, quantum spin Hall (QSH) systems are especially interesting for applications in spintronics. In this work, we study numerically the dynamics of wave packets in mercury telluride $(\mathrm{HgTe})$ quantum wells. This system presents topologically-protected spin polarized edge states (a signature of the QSH effect) when the width of the well is larger than a critical value $\left(d>d \_c \sim 6.3\right.$ $\mathrm{nm}$ ). In our simulations, we consider the evolution of Gaussian wave-packet using the BHZ Hamiltonian through the (Fourier) split-operator method. We observe the oscillatory behavior of the mean position of the packet, closely related to the effect of zitterbewegung. We also study the spin separation of the package into edge-state-like patterns. The strong dependence of this behavior with the initial conditions and the presence of electrical fields (in-plane) are also discussed.

\section{Introduction}

In the year of 2006, Bernevig, Hughes, and Zhang proposed that $\mathrm{HgTe} / \mathrm{CdTe}$ quantum wells present the Quantum Spin Hall Effect (QSHE) [1]. We study the dynamics of charge excitations in these systems. The main goal is to observe how the formation of edge-states takes place, and what other kind of phenomena appears.

In this work we investigate the time evolution of a system submitted to the BHZ Hamiltonian [1], which is given by

$$
\begin{aligned}
\widehat{H}_{B H Z}=C+ & \mathcal{M} \Gamma_{5}-\frac{\left(D+B \Gamma_{5}\right)}{\hbar^{2}}\left(\hat{p}_{x}^{2}+\hat{p}_{y}^{2}\right)+\frac{\mathcal{A} \Gamma_{1}}{\hbar} \hat{p}_{x} \\
& +\frac{\mathcal{A} \Gamma_{2}}{\hbar} \hat{p}_{y},
\end{aligned}
$$

where

$$
\Gamma_{1(2)}=\left(\begin{array}{cc}
\sigma_{1(2)} & 0 \\
0 & -\sigma_{1(2)}
\end{array}\right), \quad \Gamma_{5}=\left(\begin{array}{cc}
\sigma_{z} & 0 \\
0 & \sigma_{z}
\end{array}\right),
$$

and the $\sigma^{\prime} s$ represent are the Pauli matrices.

Since the Hamiltonian is a $4 \times 4$ matrix the state of the system is represented by a spinor $|\Psi\rangle$ of dimension 4 . Thus, in position representation we have

$$
\Psi(x, y, t)=\left(\begin{array}{l}
\psi_{E \uparrow}(x, y, t) \\
\psi_{H \uparrow}(x, y, t) \\
\psi_{E \downarrow}(x, y, t) \\
\psi_{H \downarrow}(x, y, t)
\end{array}\right) .
$$

Exploring the symmetries of the Hamiltonian is possible to split the spinor (3) in two independent two-dimensional spinors, where each one of these refers to a specific spin orientation. So, the spinor (3) may be rewritten by

$$
\begin{aligned}
& \psi_{\uparrow}(x, y, t)=\left(\begin{array}{l}
\psi_{E \uparrow}(x, y, t) \\
\psi_{H \uparrow}(x, y, t)
\end{array}\right), \\
& \psi_{\downarrow}(x, y, t)=\left(\begin{array}{l}
\psi_{E \downarrow}(x, y, t) \\
\psi_{H \downarrow}(x, y, t)
\end{array}\right) .
\end{aligned}
$$

Since the $\mathrm{BHZ}$ Hamiltonian is a block-diagonal matrix in this representation, we may define two independent Hamiltonians that act only in one of the spinors defined above. We denote these $2 \times 2$ blocks as $\widehat{H}_{\uparrow(\downarrow)}$; remembering our total Hamiltonian is the $4 \times 4$ matrix that we can write by

$$
\widehat{H}_{B H Z}=\left[\begin{array}{cc}
\widehat{H}_{\uparrow} & 0 \\
0 & \widehat{H}_{\downarrow}
\end{array}\right] .
$$

Note that, besides the spin, another degree of freedom appears in the BHZ model. We named this new feature as the pseudospin of the system. This pseudospin is associated with the composition of the spinors given by (4) and (5) in the electron-hole basis. Even though the BHZ Hamiltonian doesn't mix the spin components, the blocks of Hamiltonian matrix (6) do it with the pseudospin components.

As an example of what we mean with this definition of pseudospin, a quantum state with spin-up and pseudospin in the z-direction is given by the fourdimensional total $\mathrm{BHZ}$ spinors 


$$
\left|\uparrow, \Uparrow_{z}\right\rangle=\left(\begin{array}{c}
+1 \\
0 \\
0 \\
0
\end{array}\right), \quad\left|\uparrow, \Downarrow_{z}\right\rangle=\left(\begin{array}{c}
0 \\
+1 \\
0 \\
0
\end{array}\right) .
$$

Analogously for pseudospin in the $x$ - and $y$ directions we have, respectively

$$
\left|\uparrow, \Uparrow_{x}\right\rangle=\left(\begin{array}{c}
1 \\
+1 \\
0 \\
0
\end{array}\right), \quad\left|\uparrow, \Downarrow_{x}\right\rangle=\left(\begin{array}{c}
1 \\
-1 \\
0 \\
0
\end{array}\right),
$$

and

$$
\left|\uparrow, \Uparrow_{y}\right\rangle=\left(\begin{array}{c}
1 \\
+i \\
0 \\
0
\end{array}\right), \quad\left|\uparrow, \Downarrow_{y}\right\rangle=\left(\begin{array}{c}
1 \\
-i \\
0 \\
0
\end{array}\right) .
$$

The expressions for the correspondent spinors with spin orientation "down" can be obtained in a similar way, but with the zero and non-zero entries of spinors exchanged.

\section{Methods and Results}

The strategy adopted for the time evolution in this work is the numerical method called the FourierSplitting. This method uses the definition of timeevolution operator associated with the symmetric Strang splitting [3]. In this scenario, we may approximate the time-evolution operator by

$$
\begin{aligned}
& \widehat{U}(\delta t) \\
& \approx \exp \left[\frac{\delta t}{2}\left(-i \frac{\hat{V}}{\hbar}\right)\right] \exp \left[\delta t\left(-i \frac{\hat{T}}{\hbar}\right)\right] \exp \left[\frac{\delta t}{2}\left(-i \frac{\hat{V}}{\hbar}\right)\right] .
\end{aligned}
$$

This is possible only when we can write the Hamiltonian as a sum of a kinetic part $\widehat{T}(\widehat{\boldsymbol{p}})$ that contains all dependencies of momentum operator, and a potential part $\widehat{V}(\hat{\boldsymbol{r}})$ that, in turn, is composed by all position dependent terms of Hamiltonian. Note that the fact of $\left[x_{i}, p_{j}\right]=i \hbar \delta_{i j}$ turns (7) in an approximation.

The advantage to write the time-evolution operator in the form (7) is that one can write the exponentials intercalated with a basis transformation. That way, we can always be in a representation where the next operator to be applied is in diagonal form. Specifically, the transformation that we need is coordinate $\leftrightarrow$ momentum representation, which is reached by Fourier transform.

18th Brazilian Workshop on Semiconductor Physics (BWSP 2017)
We define our initial condition as being a twodimensional Gaussian wave-packet, multiplied by some of the 4-component spinors defined at the end of previous section. Where the Gaussian distribution is given here by

$$
\psi\left(x, y, t_{0}\right)=A \exp \left[-\frac{\left(x^{2}+y^{2}\right)}{2 \sigma}\right],
$$

where $A$ is just a normalization constant and $\sigma$ indicates how localized the packet is. All the subsequent results were obtained for wave-packets initially centered at $(0,0)$. Also, in this work we adopt the parameters for $\mathrm{CdTe} / \mathrm{HgTe} / \mathrm{CdTe}$ quantum well with width $d \approx 7 \mathrm{~nm}$ [2].

Firstly, we studied those systems in which the wave-packet center is far away from boundaries, i.e. the bulk. We modeled this situation adopting periodic boundary conditions. In this case there is no need of splitting; once the system is supposed to be free of electrical potentials, we evolve the wave function in momentum representation.

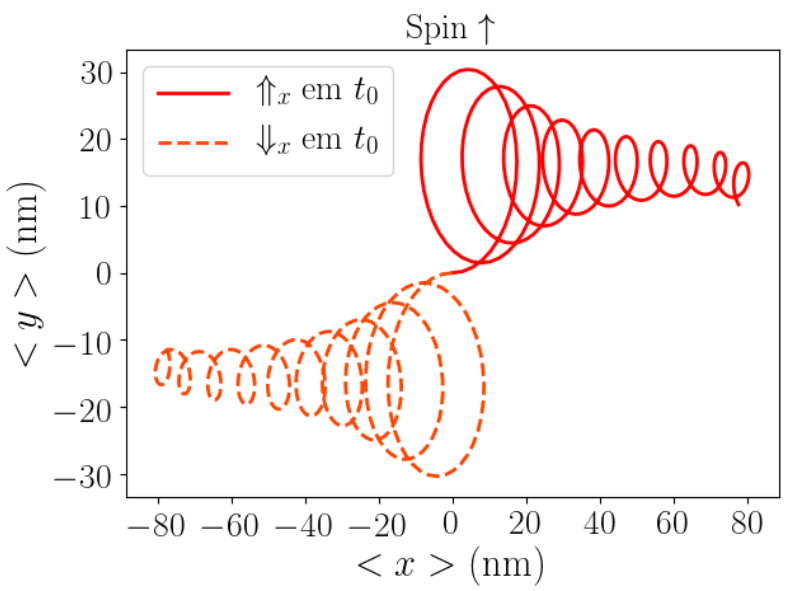

Figure 1. Trajectories of the average position of spin-up wave-packets. The continuous line represents the trajectory of pseudospin-up in " $x$ "-direction while the dashed line refers to pseudospin-down in the same direction. Here there is no external electrical potential. The initial Gaussian packet was set with $\sigma=75 \mathrm{~nm}$, the simulation run until $t=4 \mathrm{ps}$.

In the Figure 1 and 2 we see the trajectories described by the average position of the wavepacket both in systems without any potential and with pseudospin in $\mathrm{x}$-direction. Figure 1 shows the evolution of the spin-up spinors of the total spinor, while Figure 2 shows the spin-down spinors respectively. Here we use that the Hamiltonian is block diagonal to plot the spin components separately. 


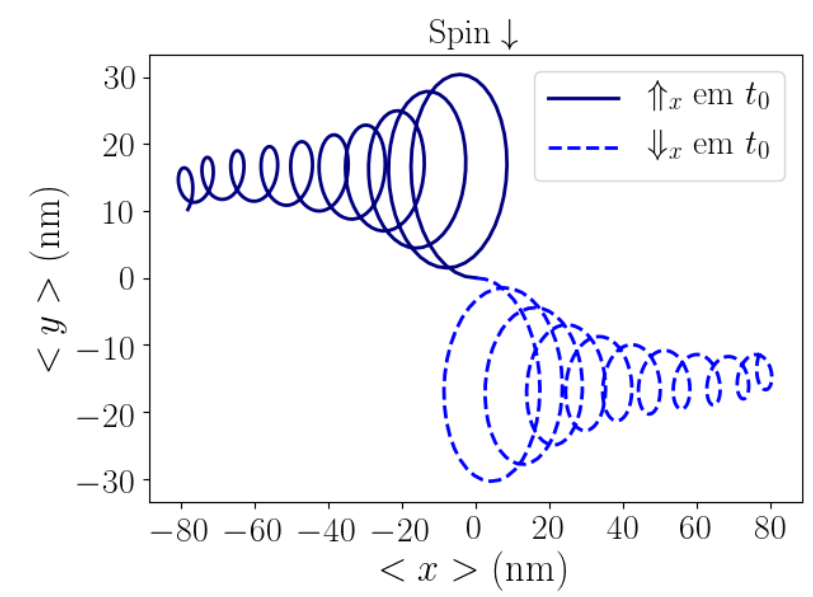

Figure 2. Trajectories of the average position of spin-down wave-packets. The continuous line represents the trajectory of pseudospin-up in the " $x$ "-direction while the dashed line refers to pseudospin-down in the same direction. Here there is no external electrical potential. The initial Gaussian packet was set with $\sigma=75 \mathrm{~nm}$, the simulation run until $t=4$ ps.

Figures 3 and 4 show the same situation with pseudospin in " $y$ "-direction, but for sake of a better visualization, in this case we divide the results by their pseudospin orientation. In Figure 3 we have spin-up and spin-down with pseudospin-up. The same for the Figure 4 but with pseudospin-down.

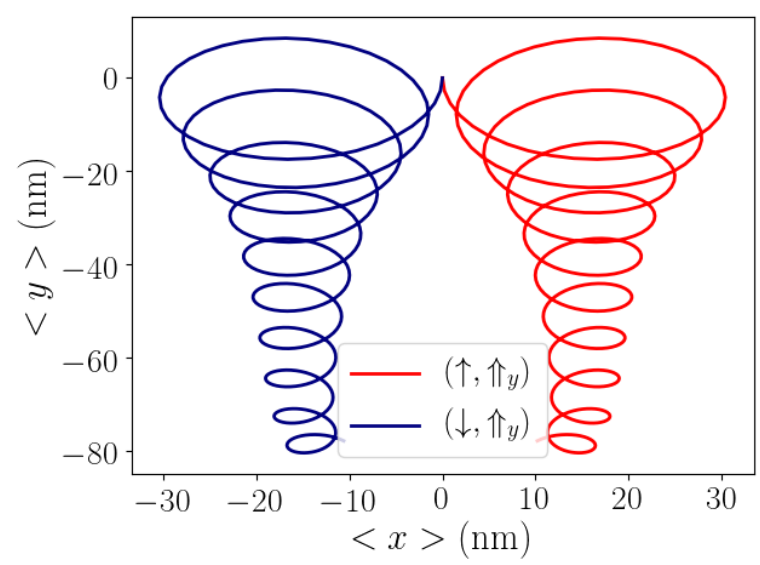

Figure 3. Trajectories of the average position of the wavepackets with pseudospin-up in the "y"-direction. The red line represents the trajectory of spin-up while the blue line refers to spin-down. Here there is no external electrical potential. The initial Gaussian packet was set with $\sigma=75$ $\mathrm{nm}$, the simulation run until $t=4 \mathrm{ps}$.

Finally, the results for pseudospin in "z"-direction are showed in the Figure 5. In this case we must include a bias potential to visualize the trembling pattern. The bigger the value of initial width of the Gaussian packet $\sigma$, the more pronounced is the separation of the trajectories. The results are in
Figure 5 we set $\sigma=300 \mathrm{~nm}$. Now we can see that setting the pseudospin orientation and "flipping" the spin, we perform a reflection across the " $x$ "-axis, On the other hand, holding the spin orientation and changing the pseudospin we observe an inversion across the origin of the average orbits. Like the other results, the wave-packets start centered at point $(0,0)$.

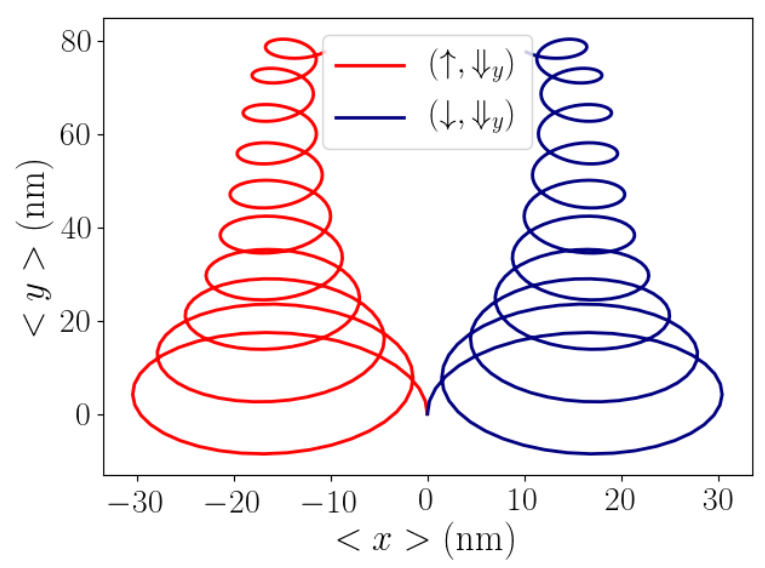

Figure 4. Trajectories of the average position of the wavepackets with pseudospin-up in the "y"-direction. The red line represents the trajectory of spin-up while the blue line refers to spin-down. Here there is no external electrical potential. The initial Gaussian packet was set with $\sigma=75$ $\mathrm{nm}$, the simulation run until $t=4 \mathrm{ps}$.

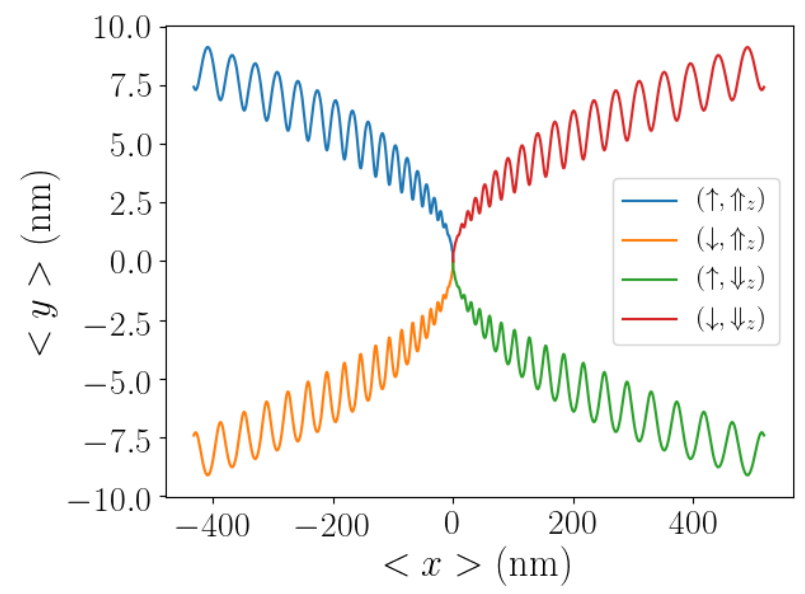

Figure 5. Trajectories of the average position of the wavepackets with pseudospin in the "z"-direction. Here we set a potential bias given by $V_{b}=10^{-3} x \mathrm{meV} \mathrm{nm}^{-1}$. The initial Gaussian packet was set with $\sigma=300 \mathrm{~nm}$, the simulation run until $t=4$ ps.

Others systems of interest are those in which the wave-packet is close to the boundaries, or at least, where the limits of the systems cannot be neglected. These cases are important in the context of topological insulators due to the research on formation and dynamics of edge states in this class 
of materials. Since we need periodic boundary conditions to use numerical Fourier transforms, we must change our strategy a little bit to simulate this systems.

To study the formation of edge states we decided to set a system in which we have periodic boundary condition on the " $x$ "-direction, but now we have the "y"-dimension limited by boundaries of hard wall type, i.e. the wave function of the system is zero outside the limits in the "y"-direction. This can be done using a hybrid formulation of the algorithm for time evolution such that we continue using the split operator in the " $x$ "-direction and a finite-differences formulation in the "y"-direction. In other words, we evolve in time a wave function represented in the $\left.\left\{k_{x}, y\right\rangle\right\}$ basis.

In Figure 6, we see the time evolution of a Gaussian wave-packet with pseudospin aligned in the " $z$ "-direction and "pointing down". In this specific polarization of pseudo spin, is possible to observe a splitting of the wave-packet, and the formation of edge states with appreciable amplitude in both limits in "y"-direction. In addition, we can see that the propagation of these edge states is spin-dependent once we obtain a mirrored image when the spinorientation is changed.

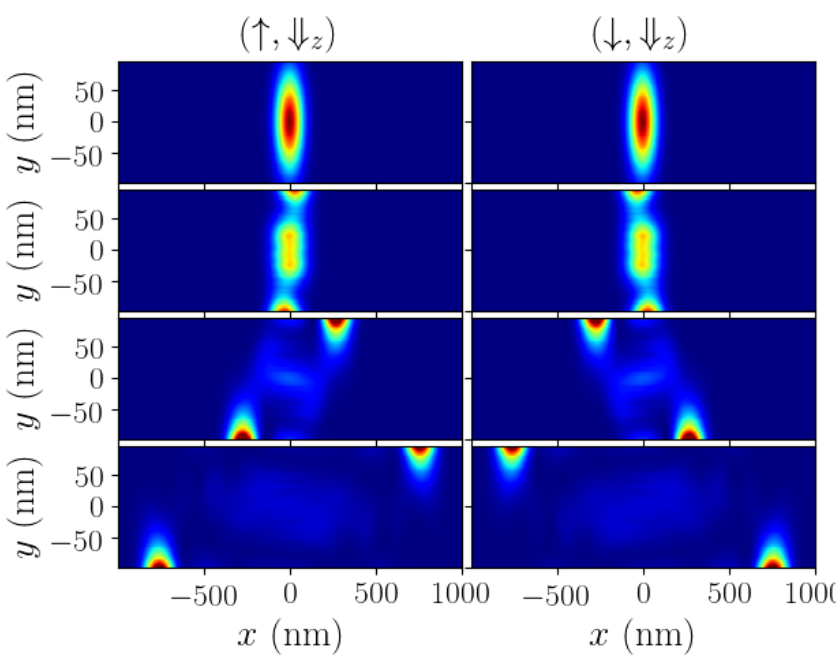

Figure 6. Evolution of the probability density of the wavepacket of a system ruled by $\mathrm{BHZ}$ Hamiltonian in the presence of hard walls in locations $y= \pm 75 \mathrm{~nm}$. Each of the columns refers to a spin orientation, and each row represents an instant of time. Specifically we have: $t_{1}=$ $0 \mathrm{ps}, t_{2}=0.1 \mathrm{ps}, t_{3}=1.0 \mathrm{ps}$, and $t_{4}=2.0 \mathrm{ps}$.
Through the results presented in last section, for pseudospin in $z$ direction, we may say that the trajectories of the average positions inside the bulk, present a pattern that have some similarities with Rashba spin-orbit coupling systems [4,5]. The trembling motion that appears on the system, perpendicular to the propagation direction, is sometimes referred as zitterbewegung (zitter). While in the case of Rashba systems, the zitter may be explained as an interference of spins states that made up the actual state of the system. In our case, of $\mathrm{BHZ}$ systems, the interplay of the pseudospins states is the root of this new kind of zitter.

On the other side, the results obtained without the application of electrical potentials and pseudospins pointed in the $\mathrm{x}$ and $\mathrm{y}$ directions, showed orbits that has a distinctive nature. The interference of the Electron-Hole states leads in this case to a formation of spiral-like trajectories with no resemblance of the known zitter in Rashba systems.

For systems with non-negligible boundaries, we observe the presence of edge states that is seemed to be equally spin- and pseudospin-dependent. The relation between the wave-packet initial width and the width of the strip ("y"-dimension), are important for the formation and stability of these edge states. For instance, a wave-packet with initial size much smaller than the strip has comparable amplitudes for bulk and edge states. Instead in the systems presented in Figure 6, we can see almost negligible bulk states amplitude.

\section{Acknowledgments}

We thank the financial support provided by $\mathrm{CNPq}$ and CAPES, and the staff and infrastructure given by University of São Paulo and Federal University of Uberlândia.

1 Bernevig, B. A.; Hughes, T. L.; Zhang, S.-C., Science, vol. 314, no 5806, p. 1757-1761, 2006.

2 Sharf, B.; Matos-Abiague, A.; and Fabian, J., Physical Review $B$, vol. 86, no 7, p. 075418, 2012.

3 Strang, G.: On the construction and comparison of difference schemes. SIAM J. Numer. Anal. 5(2), p. 506-517, 1968

4 J. Schliemann, D. Loss, and R. M. Westervelt, Phys. Rev. Lett. 94, 206801, 2005.

5 J. Schliemann, D. Loss, and R. M. Westervelt, Phys. Rev. B 73, $085323,2006$. 